Article

\title{
Quantum Bounds on Detector Efficiencies for Violating Bell Inequalities Using Semidefinite Programming
}

\author{
Alexander Sauer * and Gernot Alber \\ Institut für Angewandte Physik, Technische Universität Darmstadt, 64289 Darmstadt, Germany; \\ gernot.alber@physik.tu-darmstadt.de \\ * Correspondence: alexander.sauer@physik.tu-darmstadt.de
}

Received: 12 September 2019; Accepted: 28 December 2019; Published: 3 January 2020

check for updates

\begin{abstract}
Loophole-free violations of Bell inequalities are crucial for fundamental tests of quantum nonlocality. They are also important for future applications in quantum information processing, such as device-independent quantum key distribution. Based on a detector model which includes detector inefficiencies and dark counts, we estimate the minimal requirements on detectors needed for performing loophole-free bipartite and tripartite Bell tests. Our numerical investigation is based on a hierarchy of semidefinite programs for characterizing possible quantum correlations. We find that for bipartite setups with two measurement choices and our detector model, the optimal inequality for a Bell test is equivalent to the Clauser-Horne inequality.
\end{abstract}

Keywords: bell inequality; semidefinite programming; detector efficiency; dark counts

\section{Introduction}

Bell inequalities characterizing all possible classical correlations of local realistic theories and their violations by quantum theory have been an active field of research since Bell's original paper published in 1964 [1]. Apart from revealing characteristic quantum properties, which could also be demonstrated experimentally recently in loophole-free ways [2,3], violations of Bell inequalities are also used in quantum physics as a measure for the secrecy of quantum key distribution schemes [4,5]. Such violations can only be achieved as long as losses and inefficiencies involved in an experimental setup under investigation are sufficiently small. It is a main intention of this paper to investigate the bounds on efficiencies required for observing characteristic quantum correlations by exploring Bell inequalities in the presence of imperfect detectors.

To find the minimal requirements on these efficiencies, we treat the experimental setup as a black box, only requiring some input and yielding an output for every single measurement. For such device-independent treatments, results have already been published for several possible scenarios with different numbers of involved parties, inputs and outputs, see e.g., [6-8]. In this paper, we introduce an extension of these investigations to an error model which includes dark counts of the imperfect detectors involved.

To demonstrate the possibility of a violation of a Bell inequality there are two problems which must be solved. First, an adequate inequality must be found. To this end, one could compute and subsequently test all facets of the corresponding Bell polytope. For larger systems this is however infeasible, as the Bell polytope is typically given in its vertex representation (see Section 2.1) and finding all facets is a computationally hard problem [9]. However, one can use linear programs [10] to test if given measurement correlations lie outside the Bell polytope, thus violating some Bell inequality. The second problem is finding such correlations, which must be compatible with quantum 
theory. Nevertheless, despite repeated efforts, similarly clear conceptual restrictions as known for Bell polytopes, are not yet known for quantum correlations, see e.g., [11,12]. There are, however, mathematical restrictions, which can be put into a hierarchy of optimization programs. The resulting correlations are conjectured to represent the possible quantum correlations exactly in the limit of infinite order of the hierarchy [13]. In the following we use a combination of these techniques to find the detector parameters, which allow for a successful violation of Bell inequalities in bipartite and tripartite scenarios. This way it will not only be shown that in the absence of dark counts, our approach reproduces previous results [8], but we will also explore the detector efficiencies required for loophole-free violations of local realism in these scenarios.

\section{Results}

\subsection{Bell Polytopes, Realistic Theories and Quantum Correlations}

We summarize basic aspects concerning the theoretical description of those statistical correlations, which are typically explored by Bell experiments, within the theoretical framework of (classical) local realistic theories. The constraints imposed on these correlations within the framework of local realistic theories can be described in terms of (generalized) Bell inequalities. Even beyond the scenarios originally considered by J. S. Bell [1], for each multipartite measurement setup the structure of these correlations is described by a corresponding polytope, the so-called Bell polytope. Characteristic features of these polytopes have been explored, for example, in Refs. [9,14-16].

Let us consider a typical $N$-partite Bell-type experiment in which $N$ parties perform a joint measurement on a physical system shared between them. For this purpose, each of these parties selects a physical variable (observable) from a finite set of possible physical variables (observables), say $\mathcal{O}$. This selection can be described formally by an $N$-tuple (input) $x:=\left(x_{1}, \cdots, x_{N}\right)$ with $x_{i} \in \mathcal{O}$, $i \in\{1, \cdots, N\}$. Thus, in an $N$-partite spin experiment, for example, each party measures the spin of a many-particle system consisting of at least $N$ spin $1 / 2$ particles generally in a different direction. Depending on the choices of these physical variables each of these parties observes a measured value. All these measured values define an $N$-tuple of measured values (output) $a:=\left(a_{1}(x), \cdots, a_{N}(x)\right)$. In the following we assume that these measured values form a finite discrete set $\mathcal{A}$, i.e., $a_{i}(x) \in \mathcal{A}$ with $i \in\{1, \cdots, N\}$.

If we describe such an $\mathrm{N}$-partite Bell-type experiment within the general framework of a classical realistic theory without any further constraint, such as a locality requirement, we can distinguish between two types of theoretical descriptions, namely a deterministic one and a statistical one. In a deterministic realistic description, there exists a transfer function $F$ (or state) which relates each value of the input variable (choice of physical variable) with a unique value of the output variable (result of the measurement), i.e.,

$$
a=F(x), x \in \mathcal{O}^{N}, a \in \mathcal{A}^{N} .
$$

Within the framework of a statistical realistic theory the state of the physical system, i.e., $F$, is described by a random variable characterized by a normalized probability distribution $P(F) \geq 0$ with $\sum_{F} P(F)=1$. This probability distribution $P(F)$ generates conditional probability distributions $P(a \mid x)$ by the relation

$$
P(a \mid x)=\sum_{F} P(F) \delta(a, F(x)),
$$

with $\delta(a, F(x))=1$ for $a=F(x)$ and zero otherwise. These conditional probabilities describe the probability that the choice of the physical variables $x \in \mathcal{O}^{N}$ yields a joint measurement result $a \in \mathcal{A}^{N}$ of all the $N$ parties involved. They define a probability polytope with vertices $F$ [10] characterizing a (classical) statistical realistic theory. If the parties involved in such a Bell-type correlation experiment are pairwise space-like separated, the input of any party cannot influence the output of any other party 
due to the finite speed of light in vacuum. This locality constraint is equivalent with the requirement that each party has its own transfer function, i.e.,

$$
a=\left(F_{1}\left(x_{1}\right), \cdots, F_{N}\left(x_{N}\right)\right),
$$

so that for this locality constraint the conditional probabilities characterizing a statistical theory are of the form

$$
P(a \mid x)=\sum_{F_{1}, \cdots, F_{N}} P\left(F_{1}, \cdots, F_{N}\right) \delta\left(a_{1}, F_{1}\left(x_{1}\right)\right) \cdots \delta\left(a_{N}, F_{N}\left(x_{N}\right)\right) .
$$

These conditional probabilities define a probability polytope with vertices $\left(F_{1}, \cdots, F_{N}\right)$ characterizing a (classical) statistical local realistic theory [10].

There are two different ways to describe the probability polytope of a (classical) statistical realistic theory mathematically, either by a vertex representation or by a half-space representation. For any given experimental setup, the vertex representation of the associated probability polytope is defined in terms of the relevant transfer functions $[9,10,15]$. The half-space representation of such a probability polytope is defined by inequalities, which in the case of a locality constraint are called generalized Bell inequalities. Whereas the vertex representation can be constructed in a systematic way, in general its conversion to the corresponding half-space representation constitutes a hard mathematical problem which can typically be solved only for very small numbers of parties, numbers of observables and measurement results. In view of these problems, the exploration of generalized Bell inequalities for multipartite scenarios is still an active field of research. In the following we use the term 'Bell inequality' for any inequality, which divides the probability space in such a way that the full probability polytope of a statistical local realistic theory, i.e., its Bell polytope, is inside the half-space defined by this inequality. Of particular interest will be those inequalities, which are also facets of the relevant Bell polytope. Clearly, a violation of such a Bell inequality by a quantum system shared between $N$ parties proves that these statistical correlations cannot be described within the framework of a statistical local realistic theory. In particular, entangled quantum states may exhibit such characteristic quantum features. Apart from very simple physical systems it is still a largely open question which violations of Bell inequalities describing local realistic correlations are possible by quantum theory.

\subsection{Bell Experiments with Imperfect Detection}

In the following we explore correlations of local realistic theories in the presence of imperfections concerning the measurements involved. In particular, generalizing recent investigations we concentrate on imperfect detection of measurements in the additional presence of dark counts of detectors in bipartite and tripartite scenarios.

Putting the problem into perspective let us concentrate on one of the simplest possible generalizations with imperfect detection, namely a bipartite scenario, i.e., $N=2$, in which each party selects one of two inputs randomly, i.e., $|\mathcal{O}|=2$ and in which for each of the parties four different outcomes are possible, i.e., $|\mathcal{A}|=4$. Physically speaking these four exclusive measurement results (outputs), namely $\mathcal{A}=\{\varnothing, 1,2, D C\}$, are supposed to represent the no-detection event $(\varnothing)$, the possibly ideal measurement results 1 and 2 and the dark count event $(D C)$. To realize such a scenario, it is necessary that each party has two detectors available which can detect the exclusive measurement results 1 and 2. In a typical Bell experiment measuring polarization states of photons, for example, this would require that each party uses two photon detectors which detect two orthogonal photon polarizations. If only one photon has been sent to a party and if none of these two photon detectors of this party clicks, the event $\varnothing$, i.e., no photon present, is registered. If both detectors click a dark count $(D C)$ is taking place.

We model each of the two imperfect detectors of each party by two (statistically) independent parameters, namely $\eta$ and $\delta$. These parameters are assumed to be identical for all detectors involved. The parameter $\eta$ is the probability that a particle is detected provided a particle has been sent to the 
party. The parameter $\delta$ is the probability that a dark count is produced. Correspondingly, for each party the conditional probabilities transforming ideal outputs with potential measurement results $a^{(i d)} \in \mathcal{A}_{\text {ideal }}=\{1,2\}$ into the actually observed imperfect outputs $a \in \mathcal{A}$ are given by

$$
P_{\eta, \delta}\left(a \mid a^{(i d)}\right)=\left\{\begin{array}{ll}
(1-\eta)(1-\delta)^{2} & a=\varnothing, \text { event } A \\
(1-\eta)(1-\delta) \delta & a \neq a^{(i d)}, \text { event } B \\
\delta(1-(1-\eta)(1-\delta)) & a=D C, \text { event } C \\
(1-\delta)(1-(1-\eta)(1-\delta)) & a=a^{(i d),} \text { event } D
\end{array}\right\} .
$$

Here, $P_{\eta, \delta}\left(a \mid a^{(i d)}\right)$ is the probability to measure the result $a$ in the system with imperfect detectors, if the measurement would have resulted in the value $a^{(i d)}$ in the ideal system with perfect detection. Therefore, the four exclusive events $A, B, C, D$ have the following physical meanings:

- A: no particle is detected, and no dark count takes place in both detectors,

- $\quad B$ : no particle is detected by the detector which should have registered it in the ideal case and a dark count takes place in the other detector,

- $\quad C$ : either the particle is detected by one of the detectors and a dark count takes place in the other detector or the particle is not detected, and dark counts take place in both detectors,

- $D$ : either the particle is detected, and no dark count takes place or the particle is not detected and a dark count takes place in the detector in which the particle should have been registered.

Within this detection model the observed bipartite conditional probabilities $P(a, b \mid x, y)$ are related to the corresponding ideal (unobserved) bipartite conditional probabilities $P\left(a^{(i d)}, b^{(i d)} \mid x, y\right)$ by

$$
P(a, b \mid x, y)=\sum_{a^{(i d)}, b^{(i d)}} P_{\eta, \delta}\left(a \mid a^{(i d)}\right) P_{\eta, \delta}\left(b \mid b^{(i d)}\right) P\left(a^{(i d)}, b^{(i d)} \mid x, y\right),
$$

with $a, b \in \mathcal{A}$ and $a^{(i d)}, b^{(i d)} \in \mathcal{A}^{(i d)}$. This model for imperfect detection can be viewed as the subsequent application of a perfectly working inner box, which creates ideal correlations without errors, and of an outer box so that losses and dark counts are introduced for each party separately. Thus, a party (observer) has only access to the inputs and outputs of the outer box, while classical or quantum physics restricts the possible correlations created by the inner box.

\subsection{Required Detector Parameters for Loophole-Free Bipartite Bell Tests}

To perform a Bell test successfully, a Bell inequality must be violated using some quantum setup. Thus, we need to find a probability distribution $P$, which (a) lies outside the Bell polytope, and (b) can be achieved by quantum correlations. Here, $P$ denotes the vector of all conditional probabilities $P(a, b \mid x, y)$ characterizing the experimental setup under investigation.

To check condition (a), we use a linear program as in [10]. Given all vertices $P_{V}^{(i)}$ of the Bell polytope by evaluating all the possible combinations of transfer functions for the parties, the probabilities $P_{B}$ obtained by a convex combination of these vertices, i.e., $P_{B}=\sum_{i} w_{i} P_{V}^{(i)}$, with $w_{i} \geq 0$ and $\sum_{i} w_{i}=1$, are also part of the Bell polytope. In the bipartite case considered in this section and for ideal conditional probabilities $P\left(a^{(i d)}, b^{(i d)} \mid x, y\right)$, the conditional probabilities $P(a, b \mid x, y)$ observed with imperfect detectors are computed using the detector model. Using a convex sum of the vertices $v_{i}$, the linear program then finds the point in the Bell polytope which is closest to $P$. It returns the distance between that point and $P$ with respect to the 1-norm. For probabilities $P\left(a^{(i d)}, b^{(i d)} \mid x, y\right)$, which lie outside the Bell polytope for ideal detectors, i.e., $\eta=1$ and $\delta=0$, we can now find critical detector parameters. To do this, $\delta$ is fixed and the detection probability $\eta$ is decreased (or vice versa) until the distance of $P$ to the Bell polytope reaches 0 . Once this happens, no violation of any Bell inequality is possible with these detector parameters and the given $P\left(a^{(i d)}, b^{(i d)} \mid x, y\right)$. However, there might be some other $P\left(a^{(i d)}, b^{(i d)} \mid x, y\right)$, that, for the same detector parameters, result in a probability distribution $P$ which still is well outside the Bell polytope. 
This brings us back to condition (b), namely which $P\left(a^{(i d)}, b^{(i d)} \mid x, y\right)$ are we allowed to use when trying to find the $P_{(i d)}$ that stays outside of the Bell polytope for the worst possible detector parameters? To answer this, we use a hierarchy of semidefinite programs (SDP) [13] as described in Materials and Methods. In short, the program maximizes a linear objective function on the probabilities while following mathematical restrictions arising from quantum theoretical principles. In our case, the objective function is the left hand side of a Bell inequality of the form $\sum_{a, b, x, y} c_{a b}^{x y} P(a, b \mid x, y) \leq C$. In the following, we will choose $C=0$, which is always possible using $\sum_{a, b} P(a, b \mid x, y)=1$. For each order of the hierarchy, the SDP returns an upper bound for the quantum value on this inequality, as probabilities might be viable, which lie outside of the possible quantum correlations. The results of SDP are conjectured to be identical with the possible quantum correlations only in the limit of infinite order of the hierarchy or in special cases, e.g., a finite-dimensional quantum system and a correspondingly high order of the hierarchy, see [13].

With SDP we can find the amount of violation of a Bell inequality by quantum theory for given detector parameters and we also obtain the responsible conditional probability $P_{S D P}=$ $P\left(a^{(i d)}, b^{(i d)} \mid x, y\right)$. As the chosen Bell inequality may not be optimal, we use these probabilities to find an optimized inequality. To this end, we compute the Bell inequality which is maximally violated by $P(a, b \mid x, y)$, obtained from $P_{S D P}$ and from fixed detector parameters, by solving the linear program

$$
\begin{array}{lll}
\max _{c} & \sum_{a, b, x, y} c_{a b}^{x y} P(a, b \mid x, y) & \\
\text { s. t. } & \left|c_{a b}^{x y}\right| \leq 1 & , V=\left(v_{1} ; \ldots ; v_{n}\right) \\
& V^{T} c \leq 0 & , v_{i}=P_{V}^{(i)}: \text { i-th vertex of the Bell polytope. }
\end{array}
$$

The first constraint limits the values of the weights within the Bell inequality, as the optimal value would go to infinity otherwise. The second constraint makes sure that only inequalities are considered, which leave every vertex and thus the whole Bell polytope in the feasible half-space. In this sense, it is still a Bell inequality, although this algorithm typically does not return a facet of the polytope. To get a facet with this method, $P$ must be chosen very close to the surface of the polytope, by increasing the detector inefficiency or the dark count probability.

Using several starting points and detector efficiencies it turns out that in our model the most robust inequalities are all equivalent to the Clauser-Horne $(\mathrm{CH})$ inequality [17]

$$
P\left(a, b \mid x_{1}, y_{1}\right)+P\left(a, b \mid x_{1}, y_{2}\right)+P\left(a, b \mid x_{2}, y_{1}\right)-P\left(a, b \mid x_{2}, y_{2}\right)-P\left(a \mid x_{1}\right)-P\left(b \mid y_{1}\right) \leq 0 .
$$

They are equivalent in the sense that they only differ by a relabeling of the parties, of the inputs or outputs or by instances of $\sum_{a} P(a \mid x)=1$. Here, the no-signaling condition is used, making it possible to write $P\left(b \mid y_{1}\right)=\sum_{a} P\left(a, b \mid x_{1}, y_{1}\right)=\sum_{a} P\left(a, b \mid x_{2}, y_{1}\right)$ as the result of one party cannot depend on the input chosen by the other party. The inequality is also equivalent to the well-known Clauser-Horne-Shimony-Holt (CHSH) inequality [18] if the additional outputs created by detector errors are subsumed in one of the original outputs. This implies a lifting of the original inequality to a scenario with more possible results per measurement (here from 2 outputs to 4 ), without additional changes to the inequality [19].

The hierarchy of semidefinite programs yields improving lower bounds for necessary detector efficiencies by giving better and better upper estimates for the possible quantum correlations with each order of the hierarchy. However, this method does not tell us if and how these quantum correlations can be created experimentally. However, using the $\mathrm{CH}$ inequality, we find that the step from 2nd to 3rd order SDP does not change the results for the necessary detector parameters. This implies that the obtained probabilities, and thus detector parameters, are identical to the ones possible by quantum physics. These lower bounds on the detector parameters necessary for a successful Bell test are shown as the red crosses in Figure 1. 
To find an upper bound on the detector parameters, we take the quantum states and measurements proposed by Eberhard [7] for bipartite scenarios. These define several probability distributions $P_{E}\left(a^{(i d)}, b^{(i d)} \mid x, y\right)$. As previously described, we vary $\eta$ and $\delta$ to obtain $P_{E}(a, b \mid x, y)$ and we use our linear program to check at which point these correlations are no longer outside the Bell polytope. As there are specific instructions on how to create the needed probabilities experimentally, they definitely can be reached by quantum systems and the resulting upper bounds on the detector efficiencies are shown as the blue circles in Figure 1.

As the upper and lower bounds are identical up to numerical precision, they represent the critical detector parameters for a successful Bell test, i.e., a violation of a Bell inequality, in this bipartite scenario.

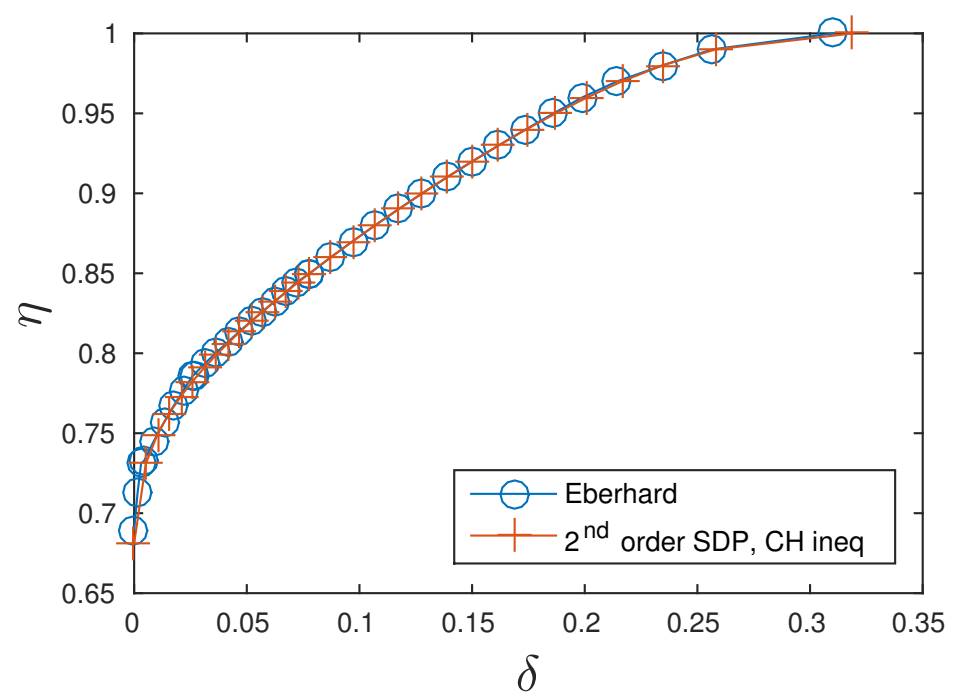

Figure 1. Necessary detector efficiency over dark count probability in the bipartite scenario. In the area below the curve, no violation of any Bell inequality is possible. If the detector efficiency $\eta$ is higher or the dark count probability $\delta$ is lower than specified by the shown critical values, quantum systems and measurements exist, which result in a violation of a Bell inequality. As the lower bounds obtained via SDP and the $\mathrm{CH}$ inequality (red crosses) are identical to the values accessible with the states and measurements given by Eberhard [7] (blue circles) up to numerical precision of the method, this bound is optimal.

\subsection{Extension to Tripartite Scenarios}

To find the critical detector parameters for a Bell experiment with three parties, we create Bell inequalities for the scenario with imperfect detectors and four outputs by lifting known inequalities for the tripartite case with dichotomic measurements. These inequalities were taken from [20], which gives a full list of the different classes of Bell inequalities for this scenario. Therefore, lifting means that the additional outputs are summed into one of the previously existing outputs as in the bipartite case with the $\mathrm{CH}$ inequality, thus making minimal changes to the Bell inequalities in order to be able to use them in a higher-dimensional scenario. This step is motivated by the previous bipartite result, where the similarly lifted $\mathrm{CHSH}$ inequality was optimal. We have also searched for better inequalities by iterative combination of a linear program and SDP as described earlier; however, this did not improve the results for the necessary detector efficiencies. In this case of three parties, there is no further change in the retrieved critical parameters for higher than 3rd order SDP, while in the scenario with 2 parties this point was reached with 2nd order SDP already. In accordance with [8], lower detector efficiencies of $\eta=0.6$ with $\delta=0$, compared to $\frac{2}{3}$ for 2 parties, are sufficient to measure the violation of a Bell inequality. In contrast to the states and measurements given by Eberhard, the states used in Larsson's proof for minimal detector efficiencies are only optimal for $\delta=0$ or $\eta=1$. These states are of the 
general form $\alpha|0\rangle+\beta|W\rangle$ with $|W\rangle$ being the multipartite W state [21]. For $\delta>0$ or $\eta<1$, they require up to $1 \%$ better detector parameters, compared to the lower bound found with SDP, to achieve a violation of a Bell inequality, even if the best possible Larsson state is chosen. The required detector efficiencies for a given dark count rate, obtained via SDP, are given in Figure 2.

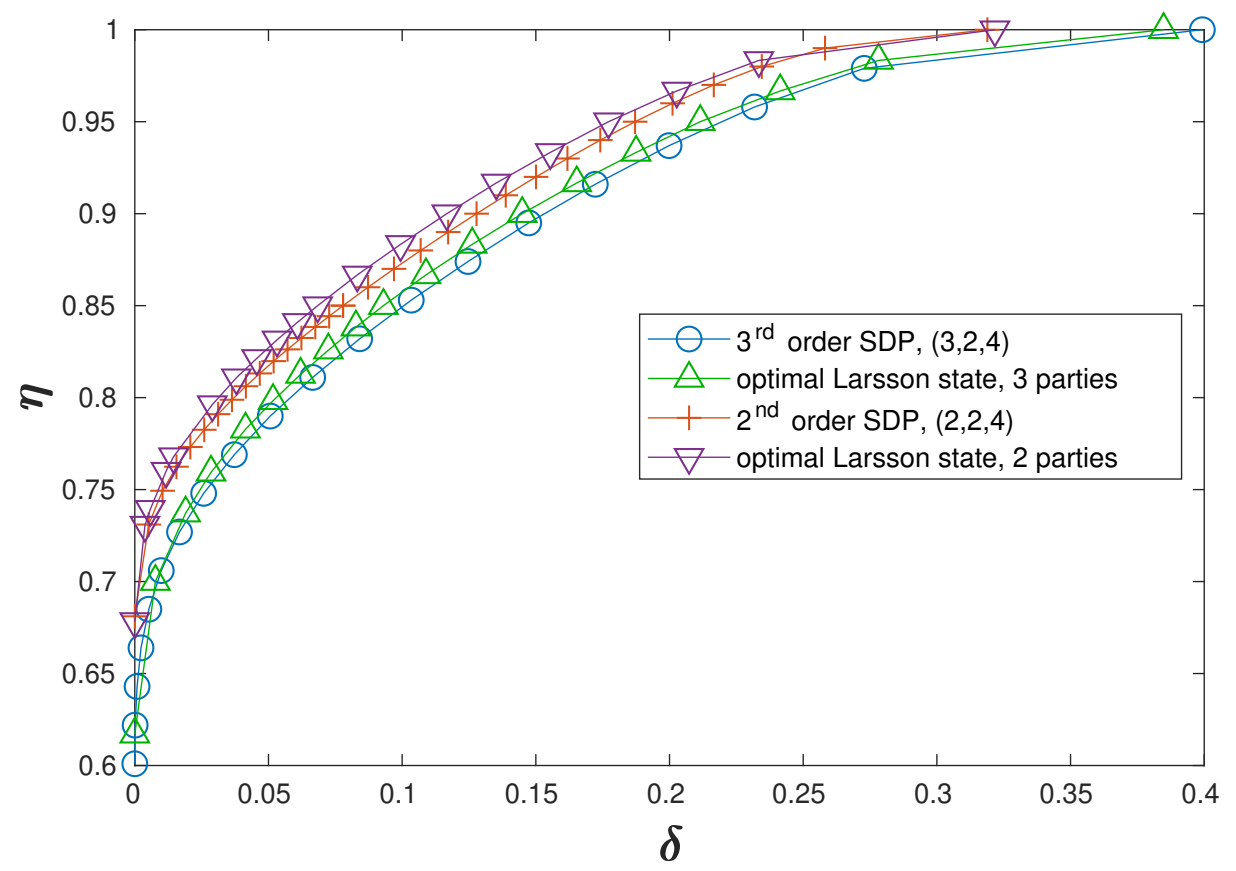

Figure 2. Necessary detector efficiencies for performing successful Bell tests with 2 and 3 parties, 2 possible measurement settings and 4 outputs each. Again, in the area below each curve no violation of any Bell inequality is possible in the corresponding scenario. For $\delta=0$ the same values for $\eta$ as in the theoretical derivation by Larsson et al. [8] are retrieved with $2 / 3$ and $3 / 5$ respectively. In the tripartite case, the requirements on the detector parameters are slightly less restrictive for all $\eta$ or $\delta$. The shown minimal detector parameters for the Larsson states cannot be reached by a single state, but are composed of the lowest bounds for different choices of parameters of the state.

\section{Discussion}

We have been able to reproduce and extend previous results on required detector parameters to observe violations of Bell inequalities by using semidefinite programming. While the Bell polytope can easily be created for any given scenario, finding the corresponding Bell inequalities is a computationally hard problem. The SDP however needs some fixed inequality as a starting point for finding a probability distribution, which maximizes its value while fulfilling all constraints of the chosen order of the hierarchy. Considering imperfect detectors and dichotomic variables as in our model, where the probability of retrieving some output only depends on the original outcomes of a perfect system and not on the chosen measurement or on the outputs of other parties, we found that it is sufficient to check lifted Bell inequalities for violation to find optimal Bell inequalities. As these results are based on our numerical analysis, a rigorous proof or a counterexample, i.e., a different inequality, which allows for worse detection efficiencies in the same general setup, has yet to be found. The SDP did not show any improvement in the results when exceeding 2nd or 3rd order for 2 and 3 parties, respectively. While we do not explicitly restrict the dimension of the underlying quantum systems, we fix the number of measurements and possible outcomes of each measurement. If all possible quantum correlations can be obtained by using low-dimensional quantum systems, e.g., qubits distributed among some parties, a correspondingly low order SDP is sufficient to obtain the possible quantum correlations, as proven in [13]. 
Our results give lower bounds on the necessary detector efficiencies for violating any Bell inequality. These bounds are general physical features, which form a basis for all two and three party cryptographic protocols that are based on entanglement. The violation can be used as a starting point to create protocols for secure key generation, see e.g., [22]. However, the computation of secure key rates would require additional assumptions about the used protocol as well as a thorough analysis of the mutual information of the involved parties and is not within the scope of this paper.

\section{Materials and Methods}

\section{Semidefinite Programming (SDP)}

To restrict our chosen ideal probabilities $P$ to probabilities, which are consistent with quantum theory, we use a hierarchy of semidefinite programs. This method has been introduced by Navascues et al. and is described in detail in [13]. Each order of the hierarchy revolves around a positive semidefinite matrix $\Gamma \succeq 0$, which is linked to the relevant conditional probabilities $P(a, b \mid x, y)$. To construct these links, first consider projective measurements with projection operators $E_{a, x}$ for all outputs of a setup. As the dimension of the quantum system is not fixed, it is always possible to use only projection operators in the model. For any pure state $|\psi\rangle$, for example, we have $P(a, b \mid x, y)=\left\langle\psi\left|E_{a, x} E_{b, y}\right| \psi\right\rangle$ and the relations

- $\quad \sum_{a} E_{a, x}=\mathbf{1}$

- $\quad E_{a, x} E_{a^{\prime}, x}=\delta_{a, a^{\prime}} E_{a, x}$

- $\quad E_{a, x}^{\dagger}=E_{a, x}$

for the projection operators involved. For order $n$ of the hierarchy, consider $S_{i}$ to be all sequences of up to $n$ of these projection operators, e.g., $E_{a, x} E_{a^{\prime}, x^{\prime}} E_{b, y}$ for $n=3$. For some fixed $|\psi\rangle$, the matrix $\Gamma$ is defined by its entries $\Gamma_{i, j}=\left\langle\psi\left|S_{i}^{\dagger} S_{j}\right| \psi\right\rangle$. Using the relations for the projection operators $E_{a, x}$, we can now determine all $K$ algebraic dependencies of the form

$$
\sum_{i, j} F_{i, j}^{(k)} \Gamma_{i, j}=\sum_{a, b, x, y} g_{a b x y}^{(k)} P(a, b \mid x, y)=: g^{(k)}(P), k \in\{1, \cdots, K\}
$$

Probabilities $P$, which cannot be obtained by a quantum system, are not able to fulfill all these conditions while keeping $\Gamma$ positive semidefinite, in the limit of $n \rightarrow \infty$. Thus, the obtained $F$ and $g$ are used to restrict $P$ in our optimization problem. To find the maximal violation of a Bell inequality $\sum_{a, b, x, y} c_{a b}^{x y} P(a, b \mid x, y) \leq C$ we use SDP in combination with the detector model. For each step of the hierarchy, the optimization problem

$$
\begin{array}{ll}
\max _{P_{(i d)}} & \sum_{a, b, x, y} c_{a b}^{x y} P(a, b \mid x, y) \\
\text { s. t. } & P(a, b \mid x, y)=\sum_{a^{(i d)}, b^{(i d)}} P_{\eta, \delta}\left(a \mid a^{(i d)}\right) P_{\eta, \delta}\left(b \mid b^{(i d)}\right) P\left(a^{(i d)} b^{(i d)} \mid x, y\right) \\
& \operatorname{tr}\left(\left(F^{(k)}\right)^{T} \Gamma\right)=g^{(k)}\left(P_{(i d)}\right) \\
& \Gamma \succeq 0,
\end{array}
$$

is solved using SeDuMi [23], a MATLAB toolbox for semidefinite optimization. The last two conditions make sure that for $P_{(i d)}$ only values that are consistent with the chosen order of the hierarchy of SDP are considered, while the program maximizes the value of the Bell inequality after applying the detector model. This procedure is repeated for higher orders of SDP until convergence, i.e., until the results are identical up to numerical precision with the results obtained with the previous order of the hierarchy. By construction, this method considers all possible correlations compatible with quantum theory without any further restrictions, such as special states or bipartite entanglement. 


\section{Conclusions}

We found that a hierarchy of semidefinite programs can be used to retrieve exact minimal requirements on detector efficiencies for loophole-free Bell tests. Although such a hierarchy of semidefinite programs is only conjectured to approach the set of quantum correlations in the limit of the order approaching infinity, in our cases only two and three steps were necessary to obtain convergence for the set of quantum correlations. This implies that these systems can always be described by a corresponding finite-dimensional quantum system. As convergence can be reached in few steps, SDP is a useful tool to find the limits of the quantum correlations for a given scenario. In the bipartite case, we could reproduce the necessary detector efficiencies given by Eberhard's results [7]. As this is an upper bound, while we produce with the hierarchy of semidefinite programs a lower bound, we were able to obtain the exact bounds on detector efficiency and dark count probability in this scenario. For the tripartite scenario with two input settings per party, we found that slightly less ideal parameters are sufficient to violate a Bell inequality. In the special case of a vanishing dark count probability, our method reproduces the lower bound which has been proven to be minimal in [8]. These bounds on detector parameters can be used in future tripartite Bell tests as they need to be fulfilled in an experiment free of the detection loophole. E.g., such an experiment might be a hub distributing quantum systems to multiple parties for quantum key distribution.

Author Contributions: Conceptualization, A.S., G.A.; methodology, A.S., G.A..; software, A.S.; validation, A.S., G.A.; investigation, A.S.; writing—original draft preparation, A.S., G.A.; writing一review and editing, A.S., G.A.; supervision, G.A. All authors have read and agreed to the published version of the manuscript.

Funding: This work was funded by the Deutsche Forschungsgemeinschaft as part of the CRC 1119 CROSSING.

Conflicts of Interest: The authors declare no conflict of interest.

\section{References}

1. Bell, J.S. On the Einstein-Podolsky-Rosen paradox. Physics 1964, 1, 195. [CrossRef]

2. Giustina, M.; Versteegh, M.A.; Wengerowsky, S.; Handsteiner, J.; Hochrainer, A.; Phelan, K.; Steinlechner, F.; Kofler, J.; Larsson, J.-A.; Abellan, C.; et al. Significant-loophole-free test of Bell's theorem with entangled photons. Phys. Rev. Lett. 2015, 115, 250401. [CrossRef] [PubMed]

3. Hensen, B.; Bernien, H.; Dréau, A.E.; Reiserer, A.; Kalb, N.; Blok, M.S.; Ruitenberg, J.; Vermeulen, R.F.; Schouten, R.N.; Abellán, C.; et al. Loophole-free Bell inequality violation using electron spins separated by 1.3 kilometres. Nature 2015, 526, 682. [CrossRef] [PubMed]

4. Ekert, A.K. Quantum cryptography based on Bell's theorem. Phys. Rev. Lett. 1991, 67, 661. [CrossRef] [PubMed]

5. Arnon-Friedman, R.; Renner, R.; Vidick, T. Simple and tight device-independent security proofs. SIAM J. Comput. 2019, 48, 181-225. [CrossRef]

6. Brunner, N.; Gisin, N.; Scarani, V.; Simon, C. Detection loophole in asymmetric Bell experiments. Phys. Rev. Lett. 2007, 98, 220403. [CrossRef] [PubMed]

7. Eberhard, P.H. Background level and counter efficiencies required for a loophole-free Einstein-PodolskyRosen experiment. Phys. Rev. A 1993, 47, R747. [CrossRef]

8. Larsson, J.-A.; Semitecolos, J. Strict detector-efficiency bounds for n-site Clauser-Horne inequalities. Phys. Rev. A 2001, 63, 022117. [CrossRef]

9. Pitowsky, I. Correlation polytopes: Their geometry and complexity. Math. Prog. 1991, 50, 395-414. [CrossRef]

10. Wilms, J.; Disser, Y.; Alber, G.; Percival, I.C. Local realism, detection efficiencies, and probability polytopes. Phys. Rev. A 2008, 78, 032116. [CrossRef]

11. Fritz, T.; Sainz, A.B.; Augusiak, R.; Brask, J.B.; Chaves, R. Leverrier, A.; Acín, A. Local orthogonality as a multipartite principle for quantum correlations. Nat. Commun. 2013, 4, 2263. [CrossRef] [PubMed]

12. Pawlowski, M.; Paterek, T.; Kaszlikowski, D.; Scarani, V.; Winter A.; Żukowski, M. Information causality as a physical principle. Nature 2009, 461, 1101. [CrossRef] [PubMed]

13. Navascués, M.; Pironio, S.; Acín, A. A convergent hierarchy of semidefinite programs characterizing the set of quantum correlations. New J. Phys. 2008, 10, 073013. [CrossRef] 
14. Brunner, N.; Cavalcanti, D.; Pironio, S.; Scarani, V.; Wehner, S. Bell nonlocality. Rev. Mod. Phys. 2014, 86, 419. [CrossRef]

15. Percival, I.C. Quantum transfer functions, weak nonlocality and relativity. Phys. Lett. A 1998, 244, 495-501. [CrossRef]

16. Wigner, E.P. On hidden variables and quantum mechanical probabilities. Am. J. Phys. 1970, 38, 1005-1009. [CrossRef]

17. Clauser, J.F.; Horne, M.A. Experimental consequences of objective local theories. Phys. Rev. D 1974, 10, 526. [CrossRef]

18. Clauser, J.F.; Horne, M.A.; Shimony, A.; Holt, R.A. Proposed experiment to test local hidden-variable theories. Phys. Rev. Lett. 1969, 23, 880-884. [CrossRef]

19. Pironio, S. Lifting Bell inequalities. J. Math. Phys. 2005, 46, 062112. [CrossRef]

20. Sliwa, C. Symmetries of the Bell correlation inequalities. arXiv 2003, arXiv:quant-ph/0305190v1. Available online: https:/ / arxiv.org/pdf/quant-ph/0305190.pdf (accessed on 20 May 2019).

21. Dür, W.; Vidal, G.; Cirac, J.I. Three qubits can be entangled in two inequivalent ways. Phys. Rev. A 2000, 62, 062314. [CrossRef]

22. Acin, A.; Gisin, N.; Masanes, L. From Bell's theorem to secure quantum key distribution. Phys. Rev. Lett. 2006, 97, 120405. [CrossRef] [PubMed]

23. Sturm, J.F. Using SeDuMi 1.02, a MATLAB toolbox for optimization over symmetric cones. Optim. Methods Softw. 1999, 11-12, 625-653. [CrossRef]

(C) 2020 by the authors. Licensee MDPI, Basel, Switzerland. This article is an open access article distributed under the terms and conditions of the Creative Commons Attribution (CC BY) license (http:/ / creativecommons.org/licenses/by/4.0/). 